SECTION 26. Radio-technique. Electronics. Telecommunications.

\author{
Alexander Andreevich Samuilov \\ Junior Researcher, \\ Laboratory of Intellectual Computer Systems of TUSUR, Russia \\ samuilovaa@gmail.com
}

\title{
COMBINED PROCEDURE OF INTERACTIVE AND AUTOMATED OPTIMIZATION SEARCH FOR VISUAL DESIGN OF CORRECTION AND MATCHING NETWORKS
}

\begin{abstract}
A new combined procedure of interactive and automated optimization search for "visual" design of correction and matching networks implementation is proposed. Using optimization techniques with a given circuit structure significantly accelerates the search.

Keywords: optimization search, correction network, matching network, "visual” design.

Citation: Samuilov AA (2014) COMBINED PROCEDURE OF INTERACTIVE AND AUTOMATED OPTIMIZATION SEARCH FOR VISUAL DESIGN OF CORRECTION AND MATCHING NETWORKS. ISJ Theoretical \& Applied Science 9 (17): 153-162. doi: http://dx.doi.org/10.15863/TAS.2014.09.17.26
\end{abstract}

УДК: 621.372.51.049.774:658.512.26:004.92

\section{КОМБИНИРОВАННАЯ ПРОЦЕДУРА ИНТЕРАКТИВНОГО И АВТОМАТИЧЕСКОГО ОПТИМИЗАЦИОННОГО ПОИСКА ПРИ «ВИЗУАЛЬНОМ» ПРОЕКТИРОВАНИИ КОРРЕКТИРУЮЩИХ И СОГЛАСУЮЩИХ ЦЕПЕЙ}

\begin{abstract}
Аннотация: Предложена реализация комбинированной процедуры интерактивного $u$ автоматического оптимизачионного поиска при «визуальном» проектировании корректируюших и согласующих иепей. Использование методов оптимизачии значительно ускоряет поиск решений при заданной структуре иепи. Первая часть статьи содержит описание комбинированной процедуры.
\end{abstract}

Ключевые слова: оптимизационный поиск, корректирующая ичепь, согласующая иепь, интерактивный поиск, «визуальное» проектирование.

Введение

В $[1,2]$ была предложена интерактивная методика «визуального» проектирования корректирующих (КЦ) и согласующих цепей (СЦ) на сосредоточенных и распределенных элементах. Данная методика позволяет синтезировать двухполюсные и реактивные четырехполюсные КЦ (СЦ) по областям допустимых значений (ОДЗ) входного иммитанса, а также решать задачу согласования активного сопротивления генератора с комплексным импедансом нагрузки (для удобства обозначим группу таких задач как задача I) - см. рис. $1 a, \sigma . \mathrm{B}[3]$ «визуальная» методика была далее развита для решения задачи согласования двух комплексных нагрузок, а также синтеза реактивных четырехполюсных цепей по ОДЗ входного и выходного иммитансов (задача II) - см. рис. 16.

Методика [1-3] позволяет решать задачи структурного синтеза КЦ (СЦ) со сравнительно небольшим количеством элементов (от 2 до 6), обеспечивает контроль как структуры, так и параметров элементов цепи. Возможность синтеза цепей по ОДЗ иммитанса разрешает эффективно использовать методику при проектировании КЦ и СЦ СВЧ транзисторных усилителей с учетом комплекса показателей [4]. Однако, так как сама по себе «визуальная» методика является интерактивной, успех проектирования в значительной мере определяется человеком, что накладывает дополнительные требования к квалификации и опыту проектировщика. 


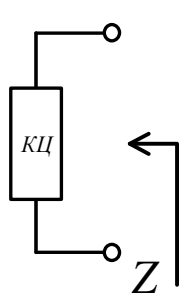

a)

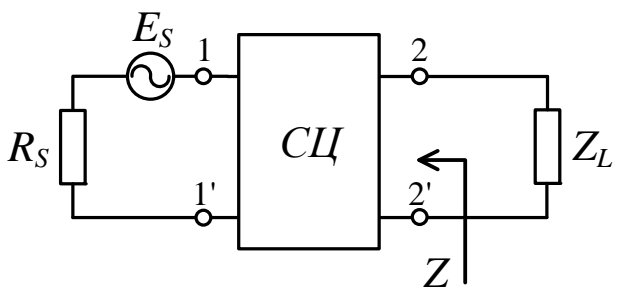

б)

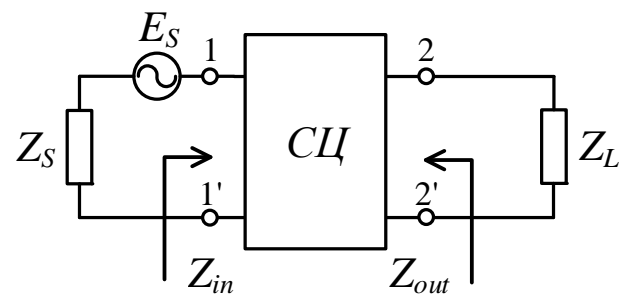

в)

Рисунок 1 - а) Задача синтеза двухполюсной КЦ по ОДЗ входного иммитанса, б) задача согласования активного сопротивления генератора с комплексным импедансом нагрузки, в) задача согласования двух комплексных нагрузок.

Другой возможный подход к перечисленным задачам состоит в параметрическом синтезе КЦ и СЦ на основе автоматического оптимизационного поиска параметров элементов. В этом случае необходимо построить целевую функцию (ЦФ), которая будет оценивать решение по взаимному расположению годографа цепи и ОДЗ. Недостатком такого подхода является то, что сложно обоснованно выбрать структуру цепи и начальные значения параметров элементов. При неудачном выборе могут быть получены неудовлетворительные локально-оптимальные решения.

В настоящей работе предлагается реализация комбинированной процедуры «визуального» проектирования КЦ и СЦ, сочетающей интерактивный и автоматический оптимизационный поиск. При автоматическом поиске пользователь визуально в реальном масштабе времени легко определяет ситуацию попадания решения в область локального оптимума. В этом случае он может, не прерывая автоматический оптимизационный поиск, интерактивными средствами изменить текущее приближение и далее продолжить автоматическое решение задачи. Комбинированная процедура позволяет значительно сократить время и повысить эффективность поиска решений. Предлагаемый алгоритм реализован в новой версии программы LOCUS.

1 Описание интерактивной методики «визуального» проектирования КЦ и СЦ. «Визуальное» проектирование КЦ (СЦ) основано на методе «областей» [5]. В случае задачи I (рис. 1a,б) вначале на каждой из выбранных частот $\omega_{k}(k=\overline{1, m})$ рабочего диапазона $\omega \in\left[\omega_{L}, \omega_{U}\right]$ строятся области допустимых значений (ОДЗ) $E\left(\omega_{k}\right)$ на плоскости входного импеданса цепи $Z$, отвечающие заданным характеристикам КЦ (СЦ) или радиоэлектронного устройства. Далее цепь синтезируется таким образом, чтобы ее импеданс $Z(\omega)$ на заданных частотах $\omega_{k}$ попадал в соответствующие ОДЗ $E\left(\omega_{k}\right)$ :

$$
Z\left(\omega_{k}\right) \in E\left(\omega_{k}\right), k=\overline{1, m} .
$$

Аналогичным образом задача (1) формулируется и в случае, если ОДЗ заданы на плоскости комплексной проводимости $Y$ или коэффициента отражения $\Gamma$.

В случае задачи II (рис. 16) ОДЗ могут строиться одновременно на плоскости входного $\left(Z_{\text {in }}\right)$ и выходного $\left(Z_{\text {out }}\right)$ импедансов (комплексных проводимостей, коэффициентов отражения) цепи.

Следует отметить, что для традиционных задач согласования двух нагрузок ОДЗ имеют вид кругов или колец и могут быть вычислены по формулам [2, 5]. Для задач проектирования КЦ и СЦ, входящих в состав СВЧ транзисторных усилителей, ОДЗ находятся с помощью специализированных программ непосредственно по требованиям к усилителю и могут иметь сложную форму [6].

Интерактивная «визуальная» процедура для решения задачи I описана в [1, 2]. Пусть $\mathbf{e}=\{R, L, C, \rho, \theta\}$ - вектор параметров элементов цепи, включающий сопротивления, индуктивности и емкости сосредоточенных элементов, волновые сопротивления и 
электрические длины отрезков линий передачи. Методика $[1,2]$ предполагает, что вектор разделяется на две части: $\mathbf{e}=\left[\mathbf{e}_{A}, \mathbf{e}_{B}\right]$. Элементы вектора $\mathbf{e}_{A}$ (так называемые управляемые элементы цепи) выбирает сам проектировщик, элементы вектора $\mathbf{e}_{B}$ зависят от $\mathbf{e}_{A}$ и определяются автоматически.

ОДЗ $E\left(\omega_{k}\right)$ визуально отображаются на плоскости импеданса $Z$ (или проводимости Y). На первом шаге методики [1, 2] выбирается структура КЦ (СЦ) путем сравнения расположения ОДЗ с формой годографов иммитанса типовых цепей, содержащихся в библиотеке.

На втором шаге в рабочем диапазоне частот $\left[\omega_{L}, \omega_{U}\right]$ выбирается опорная частота $\omega_{\text {ref }}$, а также с помощью «мыши» - опорный импеданс (опорная точка) $Z_{\text {ref }}$ в пределах ОДЗ $E\left(\omega_{\text {ref }}\right)$. Годограф импеданса $Z(\omega)$ цепи с найденной структурой для начальных значений вектора $\mathbf{e}_{A}$ также отображается на плоскости Z. Далее проектировщик в интерактивном режиме с помощью тюнеров выбирает значения управляемых элементов $\mathbf{e}_{A}$ таким образом, чтобы точки годографа $Z(\omega)$ на всех частотах $\omega_{k}$ попали в соответствующие ОДЗ $E\left(\omega_{k}\right)$. Элементы вектора $\mathbf{e}_{B}$ при этом постоянно «следят» за элементами $\mathbf{e}_{A}$, они автоматически рассчитываются в режиме реального времени из условия, что годограф $Z(\omega)$ на частоте $\omega_{\text {ref }}$ должен проходить через опорную точку $Z_{\text {ref }}$. C указанной целью решается система уравнений:

$$
\left\{\begin{array}{l}
\operatorname{Re} Z\left(\omega_{r e f}, \mathbf{e}_{A}, \mathbf{e}_{B}\right)=\operatorname{Re} Z_{r e f} \\
\operatorname{ImZ}\left(\omega_{r e f}, \mathbf{e}_{A}, \mathbf{e}_{B}\right)=\operatorname{Im} Z_{r e f},
\end{array}\right.
$$

Интерактивная «визуальная» методика для решения задачи II предложена в [3]. Если цепь синтезируется по произвольным ОДЗ, заданным на плоскостях входного и выходного иммитансов, то задачи вида (1) должны быть решены одновременно для обоих иммитансов (в случае согласования двух комплексных нагрузок достаточно решить задачу (1) для одного из иммитансов).

Проектируемая КЦ или СЦ состоит из реактивной цепи-«ядра» и подключенных к ней дополнительных управляемых реактивных (сосредоточенных или распределенных) элементов. Цепь-«ядро» содержит два реактивных сосредоточенных элемента либо отрезок линии передачи, которые характеризуются вектором $\mathbf{e}_{\text {в }}$. Дополнительные управляемые элементы характеризуются вектором $\mathbf{e}_{A}$, они могут быть включены последовательно или параллельно на входе и (или) выходе цепи-«ядра». При этом пользователь сам указывает либо выбирает в ходе проектирования способ включения (последовательный или параллельный) и тип (индуктивность или емкость) дополнительного управляемого элемента.

После отображения на одной из плоскостей ОДЗ $E\left(\omega_{k}\right)$ пользователь выбирает опорную частоту $\omega_{\text {ref }}$ и опорный импеданс $Z_{\text {ref }}$ в пределах ОДЗ $E\left(\omega_{\text {ref }}\right)$. Далее из этих условий в результате решения системы уравнений [3] автоматически определяются структура и параметры $\mathbf{e}_{B}$ реактивной цепи-«ядра». Процедура «визуального» проектирования состоит в интерактивном подборе значений управляемых элементов $\mathbf{e}_{A} \mathrm{c}$ помощью тюнеров таким образом, чтобы годограф входного и (или) выходного иммитансов цепи на частотах $\omega_{k}$ попал в соответствующие ОДЗ $E\left(\omega_{k}\right)$. Как и ранее, элементы вектора $\mathbf{e}_{B}$ «следят» за элементами $\mathbf{e}_{A}$ и автоматически рассчитываются в режиме реального времени. Для облегчения выбора структуры цепи задача может решаться сразу для нескольких цепей, отличающихся «ядром», а также типом и способом включения 
дополнительных управляемых элементов (одновременно могут отображаться годографы до четырех цепей).

При достаточно большом числе элементов КЦ или СЦ (больше 3-4) задача интерактивного «визуального» определения значений этих элементов с помощью «мыши» и тюнеров является достаточно сложной и требует от проектировщика специальных навыков. Идея настоящей работы состоит в одновременном использовании интерактивной «визуальной» процедуры и алгоритма автоматического оптимизационного поиска при нахождении значений элементов цепи. Выбор структуры цепи так же, как и в [1-3], остается за проектировщиком.

2 Решение задачи оптимизационного поиска элементов цепи по ОД3 иммитанса. Задача автоматического поиска значений элементов цепи с заданной структурой по ОДЗ иммитанса в общем случае сводится к задаче многомерной оптимизации:

$$
F(\mathbf{e}) \rightarrow \text { opt; } \mathbf{e} \in D_{\mathrm{e}} ; D_{\mathrm{e}}=\left\{\mathbf{e} \mid e_{i}^{-} \leq e_{i} \leq e_{i}^{+}\right\}, i=\overline{1, n}
$$

где opt - критерий оптимальности (min или max), e - вектор варьируемых параметров элементов цепи, $n$ - количество варьируемых параметров, $F(\mathbf{e})$ - целевая функция (ЦФ), $D_{\text {e }}$ - область, определяющая допустимые значения элементов вектора е.

Построение ЦФ. Специфика оптимизационной задачи состоит в ее «графической» сути - см. (1): необходимо найти вектор е, при котором годограф импеданса $Z(\omega, \mathbf{e})$ цепи на частотах $\omega_{k}$ попадает в ОДЗ $E\left(\omega_{k}\right)$. Таким образом, требуется построить подходящие ЦФ, оценивающие взаимное расположение годографа цепи и ОДЗ.

Рассматриваемые ниже ЦФ основаны на вычислении расстояния $S_{k}(k=\overline{1, m})$ от точки годографа $Z\left(\omega_{k}\right)$ до ближайшей границы соответствующей области $E\left(\omega_{k}\right)$. Расстояние полагается отрицательным, если точка $Z\left(\omega_{k}\right)$ находится внутри области $E\left(\omega_{k}\right)$ и положительным - если вне этой области:

$$
S_{k}=\left\{\begin{array}{r}
l, Z\left(\omega_{k}\right) \notin E\left(\omega_{k}\right) ; \\
-l, Z\left(\omega_{k}\right) \in E\left(\omega_{k}\right)
\end{array}\right.
$$

где $l$ - минимальное расстояние от точки годографа $Z\left(\omega_{k}\right)$ до границы соответствующей ОДЗ $Е\left(\omega_{k}\right)$.

По известным расстояниям $S_{k}$ можно построить несколько различных ЦФ для оценки степени выполнения условий (1). Приведем вид предлагаемых нами ЦФ (весовые коэффициенты $P_{k}$ в формулах (5)-(8) определяют важность удовлетворения требований (1) на каждой из частот $\left.\omega_{k}\right)$.

1) Минимаксная целевая функция - данная функция стремится минимизировать максимальное взвешенное расстояние до области:

$$
F=\max \left(P_{1} \cdot S_{1}, P_{2} \cdot S_{2}, \ldots, P_{m} \cdot S_{m}\right)
$$

Минимаксная целевая функция позволяет обеспечить равное расстояние от годографа до границ ОДЗ на всех частотах $\omega_{k}$. Для нахождения оптимального решения необходимо минимизировать значение функции (5).

2) Среднестепенная целевая функиия - сумма взвешенных расстояний до области, возведенных в заданную (нечетную) степень $q$ :

$$
F=\sum_{k=1}^{m} P_{k} \cdot S_{k}^{q}, q=1,3,5, \ldots
$$

где $q$ - показатель степени. Для нахождения оптимального решения необходимо минимизировать значение функции (6). 
3) R-функциия - это действительная функция непрерывных действительных аргументов, знак которой определяется только знаками аргументов и не зависит от абсолютных величин последних. $R$-функции, введенные В.Л. Рвачевым $[7,8]$, представляют собой аналитические эквиваленты логических (булевых) функций. функций:

Для оценки качества решения нами были использованы следующие виды $R$ -

а) симметричная

$$
F(S)=\sum_{k=1}^{m}(-1)^{v} \cdot\left(P_{k} \cdot S_{k}\right)^{v} \cdot\left(P_{k} \cdot S_{k}-\left|P_{k} \cdot S_{k}\right|\right)+\prod_{k=1}^{m}\left(P_{k} \cdot S_{k}\right)^{v} \cdot\left(P_{k} \cdot S_{k}+\left|P_{k} \cdot S_{k}\right|\right)
$$

где $v$ - коэффициент дифференцируемости, $v=1,2,3 \ldots$;

б) несимметричная

$$
F=F_{m}
$$

где

$$
\left\{\begin{array}{l}
F_{1}=P_{1} \cdot S_{1} \\
F_{k}=\frac{P_{k}}{1+\alpha}\left(F_{k-1}+S_{k}-\sqrt{F_{k-1}^{2}+S_{k}^{2}-2 \cdot \alpha \cdot F_{k-1} \cdot S_{k}}\right), k=\overline{1, m},
\end{array}\right.
$$

$\alpha-$ коэффициент гладкости, $-1 \leq \alpha<1$.

Для нахождения оптимального решения необходимо максимизировать значение $R$ функций (7) и (8).

Таким образом, рассмотренные ЦФ сконструированы таким образом, что при их оптимизации годограф цепи будет «стремиться» попасть внутрь каждой из ОДЗ $E\left(\omega_{k}\right)$ как можно ближе к ее центру.

В качестве методов автоматического поиска нами выбраны два распространенных сравнительно простых метода параметрической оптимизации, один из которых является случайным, а другой - детерминированным. Оба метода относятся к так называемым прямым методам, т.е. не требуют вычисления частных производных ЦФ. Данные литературы, а также проведенные нами эксперименты показывают, что эти методы также весьма надежны и малочувствительны к выбору настроечных параметров.

Метод дифференциальной эволющии [9] относится к эволюционным методам оптимизации, который предполагает создание множества начальных решений и их случайное «смешивание». При этом каждое подобное решение называется особью, а весь набор исходных решений - популяцией. На каждой итерации в общем случае происходит определение лучших особей в популяции - решений с лучшим значением ЦФ - и создание на их основе новых решений. Новые решения добавляются в популяцию, а самые худшие особи удаляются.

Симплекс-метод Нелдера-Мида [10] относится к детерминированным методам оптимизации. Суть его заключается в определении в пространстве возможных решений некоторого многогранника (симплекса) с числом вершин $n+1$, где $n-$ количество варьируемых параметров. На каждой итерации производится расчет значений ЦФ для каждой вершины многогранника, а также определяются лучшее и худшее решения. Целью каждой итерации является улучшение худшего решения на вершине симплекса. Новое решение рассчитывается с помощью операций отражения, растяжения, сжатия и редукции. В результате начальный многогранник с каждой последующей итерацией будет перемещаться в направлении локального минимума целевой функции, либо сжиматься, если оптимум находится внутри симплекса.

Проверка принадлежности вектора е области $D_{\text {e }}$ происходит следующим образом: если какой-либо элемент $e_{i}$ вектора е выходит за пределы области допустимых значений, то значение $e_{i}$ смещается в область допустимых значений по формуле: 


$$
e_{i}^{\prime}=\left\{\begin{array}{l}
e_{i}^{-}+0,1 \cdot\left(e_{i}^{-}-e_{i}\right), e_{i}<e_{i}^{-} ; \\
e_{i}^{+}-0,1 \cdot\left(e_{i}-e_{i}^{+}\right), e_{i}>e_{i}^{+},
\end{array}\right.
$$

где $e_{i}^{\prime}-$ новое значение параметра цепи, $e_{i}^{-}$и $e_{i}^{+}-$нижняя и верхняя граница области допустимых значений параметра $e_{i}$.

Реализация автоматического поиска. При «визуальном» проектировании автоматический поиск работает в двух режимах:

1) в режиме оптимизации с фиксированной опорной точкой (оптимизируются только параметры управляемых элементов цепи $\mathbf{e}_{A}$, a остальные параметры $\mathbf{e}_{B}$ рассчитываются путем решения системы уравнений из условия прохождения годографа через опорную точку);

2) в режиме оптимизации всех параметров (в вектор е входят параметры всех элементов цепи, годограф в общем случае не проходит через опорную точку).

Первый режим является основным, так как он позволяет захватывать и передвигать в нужное место с помощью «мыши» опорную точку, управляя формой годографа. Второй режим является вспомогательным и может использоваться при окончательной оптимизации параметров цепи.

При первом запуске автоматического оптимизационного поиска в качестве нулевого приближения для вектора $\mathbf{e}_{\text {A }}$ (или е) используются исходные значения элементов цепи, устанавливаемые самим пользователем. При следующих запусках оптимизационного поиска в качестве начального приближения для $\mathbf{e}_{\text {A }}$ или е берутся текущие величины параметров элементов, полученные с помощью интерактивной «визуальной» процедуры.

Особенностью автоматического поиска при «визуальном» проектировании является то, что остановка процесса оптимизации осуществляется самим пользователем. Это происходит, либо когда найдено решение (все точки годографа попали в соответствующие ОДЗ), либо когда алгоритм «скатывается» к локально-оптимальному решению, не удовлетворяющему проектировщика (часть точек годографа находятся вне ОДЗ). В последнем случае проектировщик «визуальными» средствами изменяет начальное приближение и далее вновь проводит оптимизационный поиск.

3 Организация одновременного интерактивного и автоматического поиска при «визуальном» проектировании КЦ и СЦ. Параллельная реализация режимов интерактивного и автоматического поиска решений в программах автоматизированного проектирования возможна путем построения многопоточного приложения.

Исполняемая программа с точки зрения операционной системы представляется в виде так называемого процесса, который состоит из адресного пространства, содержащего в себе код и данные всех модулей. Процессы инертны; для того, чтобы процесс что-нибудь выполнял, в нем должен существовать поток. Именно потоки определяют последовательность исполнения кода, содержащегося в адресном пространстве процесса. Один процесс может владеть несколькими потоками, и тогда они одновременно исполняют код в адресном пространстве процесса. Например, при моделировании цепей потокам разрешено исполнять один и тот же алгоритм анализа и манипулировать одними и теми же значениями вектора е.

Чтобы все эти потоки работали параллельно, операционная система отводит каждому из них определенное процессорное время. При выделении потокам поочередно квантов времени создается иллюзия одновременного выполнения потоков.

Путем реализации многопоточного приложения может быть осуществлено интерактивное воздействие пользователя на ход решения оптимизационной задачи в режиме реального времени (рис. 2). В каждом процессе всегда существует хотя бы один 
(основной) поток, который ведет обработку системных сообщений и обеспечивает взаимодействие с пользователем (поток 0). Автоматизированный поиск решений осуществляется в другом потоке (поток 1). С точки зрения исполнения программы действия пользователя, влияющие на решение задачи, можно расценивать как работу еще одного оптимизирующего потока (поток 2). Оптимизирующим методом такого потока является мышление пользователя.

Пусть на $i$-ом шаге комбинированного поиска, исходя из начального приближения $\mathbf{e}_{0}^{(i)}$, потоком 1 получено оптимальное решение $\mathbf{e}_{\text {opt }}^{(i)}$, не удовлетворяющее заданным требованиям (1). Тогда пользователь, вмешиваясь в работу основного потока, на следующем (i+1)-ом шаге может всегда установить в потоке 2 то значение вектора параметров $\mathbf{e}_{0}^{(i+1)}$, которое он считает нужным. При этом следующее начальное приближение $\mathbf{e}_{0}^{(i+1)}$ выбирается в интерактивном режиме, как правило, с учетом ранее полученного решения $\mathbf{e}_{\text {opt }}^{(i)}$ путем определенной модификации последнего и, следовательно, оно чаще всего лучше, чем $\mathbf{e}_{0}^{(i)}$.

Таким образом, параллельно с работой алгоритма автоматической оптимизации становится возможным интерактивное воздействие пользователя в реальном времени на ход решения оптимизационной задачи. Этот процесс может быть представлен следующим образом (см. рис. 2):

$$
\ldots \mathbf{e}_{0}^{(i)} \underset{\text { поток } 1}{\longrightarrow} \mathbf{e}_{\text {opt }}^{(i)} \underset{\text { поток } 2}{\longrightarrow} \mathbf{e}_{0}^{(i+1)} \ldots
$$

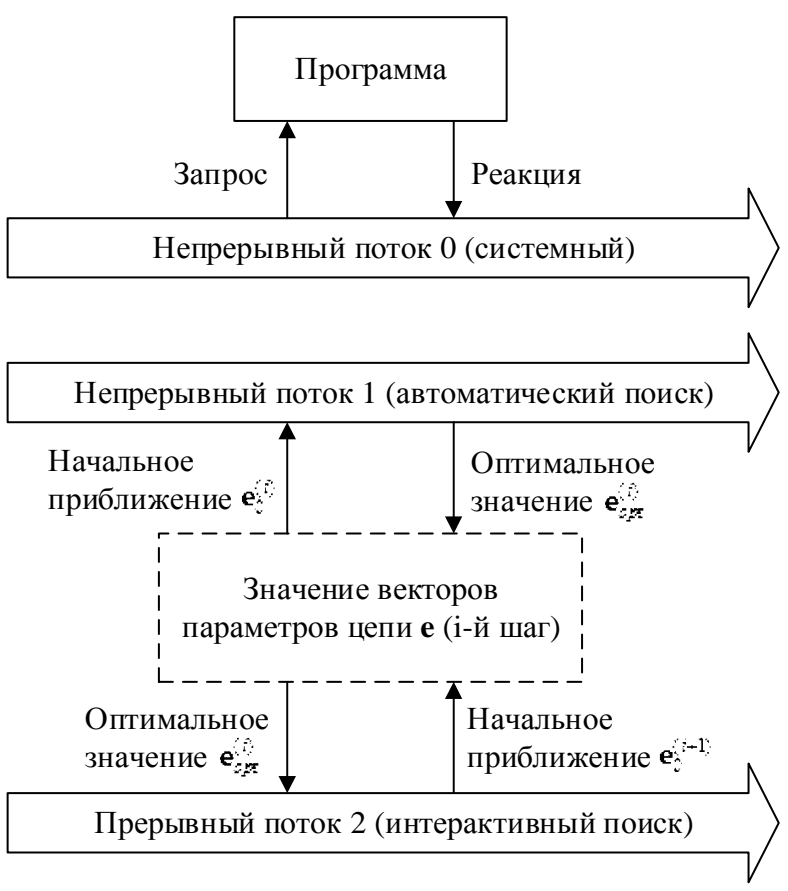

\section{Рисунок 2 - Схема интерактивного воздействия пользователя на ход решения оптимизационной задачи.}

Реализация одновременного интерактивного и автоматического поиска особенно эффективна в программах, основанных на «визуальных» процедурах решения задач, в частности, в программах визуального проектирования. Это объясняется тем, что пользователь может визуально в режиме реального времени наблюдать ход автоматического решения задачи, оценивать качество решения и выявить ситуацию, когда решение попадает в область локального оптимума. В этом случае он имеет возможность, не 
прерывая автоматический поиск, интерактивными средствами оперативно изменить текущее приближение и далее продолжить решение задачи. Таким образом, пользователь получает в свое распоряжение инструмент, который позволяет повысить эффективность поиска решений, а также предоставляет новые возможности для исследования проектных задач.

Опишем предлагаемую комбинированную процедуру интерактивного и автоматического оптимизационного поиска при «визуальном» проектировании КЦ и СЦ более подробно.

На первом этапе выбираем структуру цепи. В случае задачи I [1, 2] структура цепи выбирается из библиотеки путем сравнения расположения ОДЗ на плоскости импеданса (или комплексной проводимости) с формой годографа для типовых цепей. Пользователь в выбранной цепи назначает управляемые элементы. В случае задачи II [3] пользователь выбирает число, способ включения и тип дополнительных управляемых (сосредоточенных или распределенных) элементов цепи.

После этого программа вычисляет начальные значения параметров управляемых элементов цепи $\mathbf{e}_{A}$.

Далее среди частот $\omega_{k}$ выбираем опорную частоту $\omega_{r e f}$ и в пределах соответствующей области $E\left(\omega_{\text {ref }}\right)$ на плоскости $Z$ указываем с помощью «мыши» опорное значение входного импеданса цепи на этой частоте: $Z\left(\omega_{r e f}\right) \in E\left(\omega_{\text {ref }}\right)$. В качестве опорной частоты целесообразно выбрать частоту, на которой ОДЗ имеет наименьшие размеры. В этом случае легче исследовать влияние выбора опорной точки на форму годографа. Если размеры ОДЗ на всех частотах примерно одинаковы, за опорную можно взять нижнюю или верхнюю граничную частоту рабочего диапазона.

Следующим шагом является выбор процедуры проектирования КЦ или СЦ - чисто «визуальной» или комбинированной. В случае только «визуального» поиска проектировщик сам подбирает значения параметров управляемых элементов цепи с помощью тюнеров, а остальные элементы рассчитываются в результате решения системы уравнений.

В случае комбинированного оптимизационного поиска пользователь выбирает метод оптимизации, целевую функции и режим работы оптимизационного поиска - с фиксированной опорной точкой или при оптимизации всех параметров, а также задает диапазоны варьирования параметров каждого элемента цепи. Для того, чтобы определить важность удовлетворения требований (1) на каждой из частот $\omega_{k}$, вводятся весовые коэффициенты $P_{k}$.

Далее происходит запуск процесса автоматической оптимизации. В случае режима работы с фиксированной опорной точкой в качестве начального приближения для оптимизатора используются текущие значения параметров управляемых элементов цепи $\mathbf{e}_{A}$ . На очередной итерации процесса оптимизации выбираются определенные параметры управляемых элементов цепи $\mathbf{e}_{A}$. Далее происходит определение $\mathbf{e}_{B}$ путем решения системы уравнений (2), расчет и отображение годографа $Z\left(\omega_{k}\right)$ на плоскости $Z$. После этого, для каждой из частот $\omega_{k}$ рассчитываются расстояния $S_{k}$ от точки годографа $Z\left(\omega_{k}\right)$ до ближайшей границы соответствующей области $E\left(\omega_{k}\right)$, далее по одной из формул (5)-(8) в зависимости от выбранной ЦФ оценивается текущее решение. Основываясь на этой оценке, оптимизатор изменяет значение вектора элементов $\mathbf{e}_{A}$, и процесс повторяется сначала.

После запуска процесса оптимизации пользователь в режиме реального времени наблюдает изменение формы годографа на плоскости $Z$ и оценивает попадание точек годографа $Z\left(\omega_{k}\right)$ в соответствующие ОДЗ $E\left(\omega_{k}\right)$. Процесс автоматического поиска 
решений происходит параллельно с интерактивными действиями пользователя в этот момент. Это означает, что пользователь может вмешиваться в работу оптимизатора, перемещая точки годографа с помощью «мыши», либо изменяя параметры управляемых элементов цепи с помощью тюнеров, и устанавливать (фиксировать) такие значения элементов цепи, которые считает нужными.

Наблюдая ход автоматического поиска решения задачи, пользователь может визуально в режиме реального времени выявить ситуацию, когда оптимизационный алгоритм попадает в область локального оптимума - при этом форма годографа перестает изменяться, но одна или несколько точек годографа находятся за пределами ОДЗ. В таком случае он имеет возможность, не прерывая автоматический поиск, с помощью мыши или тюнеров изменить положение годографа и далее продолжить автоматическое решение задачи.

Остановка процесса оптимизации происходит самим пользователем - либо когда найдено решение (все точки годографа попали в соответствующие ОДЗ), либо когда невозможно найти решение с заданной структурой цепи и для продолжения синтеза необходимо выбрать другую ее структуру.

Предложенная комбинированная процедура проектирования КЦ (СЦ) реализована в новой версии программы Locus.

Заключение. Визуализация процесса оптимизации и реализация комбинированной процедуры интерактивного и автоматического оптимизационного поиска при «визуальном» проектировании КЦ и СЦ, описанная в данной работе, расширяет возможности при решении задач оптимизации:

1) позволяет человеку вмешиваться и корректировать ход решения;

2) разрешает использовать несколько методов оптимизации;

3) дает возможность для одних и тех же методов оптимизации построить разные ЦФ;

4) ускоряет поиск решения;

5) способствует нахождению глобального оптимума.

\section{References:}

1. Babak LI, Cherkashin MV (2001) Interactive «visual» design of matching and compensation networks for microwave active circuits. IEEE MTT-S International Microwave Symposium Digest. Phoenix. AZ, pp.2095-2098.

2. Babak LI, Cherkashin MV, Zaytsev DA (2006) «Vizual'noe» proektirovanie korrektiruyushchikh i soglasuyushchikh tsepey poluprovodnikovykh SVCh-ustroystv. Ch. 1. Opisanie protsedury proektirovaniya. sbornik dokladov TUSUR, No. 6 (14); izdatel'stvo TUSUR, pp.11-23.

3. Samuilov AA, Cherkashin MV, Babak LI (2013) Metodika «vizual'nogo» proektirovaniya tsepey na sosredotochennykh elementakh dlya shirokopolosnogo soglasovaniya dvukh kompleksnykh nagruzok. sbornik dokladov TUSUR, No.2 (28), izdatel'stvo TUSUR, pp.3039.

4. Babak LI (2001) Decomposition synthesis approach to design of RF and microwave active circuits. IEEE MTT-S International Microwave Symposium Digest. Phoenix. AZ, pp. 11671170.

5. Babak LI (1995) Sintez soglasuyushchikh tsepey i tsepey svyazi tranzistornykh shirokopolosnykh usiliteley po oblastyam immitansa. Radiotekhnika i elektronika. T. 40, No.10, pp. 1550-1560.

6. Babak LI, Cherkashin MV, Polyakov AYu, Bodunov KS, Dyagilev AV (2005) Programmy "vizual'nogo" proektirovaniya tranzistornykh SVCh usiliteley. 15-ya Mezhd. Krymskaya konf. 
"SVCh-tekhnika i telekommunikatsionnye tekhnologii" (KryMiKo'2005). Sevastopol' : Veber, T. 2, pp. 425-426.

7. Rvachev VL (1967) Geometricheskie prilozheniya algebry logiki. Kiev: Tekhnika, 212.

8. Rvachev VL (1982) Teoriya R-funktsiy i nekotorye ee prilozheniya. Kiev: Naukova dumka, 552.

9. Storn R, Price K (1997) Differential Evolution - A Simple and Efficient Heuristic for Global Optimization over Continuous Spaces. Journal of Global Optimization, No.11, pp. 341-359.

10. Khimmel'blau D (1975) Prikladnoe nelineynoe programmirovanie. Pod red. ML Bykhovskogo. Moscow: Izdatel'stvo «Mir», 536. 